\title{
REA_17 - Utility of oral fluid samples to determine hepatitis B virus genotypes, mutations and phylogenetic analysis
}

Livia Melo Villar ${ }^{1 *}$; Barbara Vieira do Lago ${ }^{1}$; Cristianne Sousa Bezerra ${ }^{2}$; Ana Carolina da Fonseca Mendonça ${ }^{1}$; Leticia Cancella Nabuco ${ }^{3}$; Cristiane Alves Villela-Nogueira ${ }^{3}$; Moyra Machado Portilho ${ }^{4}$.

${ }^{1}$ Fiocruz/IOC;

${ }^{2}$ Universidade Federal do Ceará;

${ }^{3} \mathrm{UFRJ} / \mathrm{HUCFF}$;

${ }^{4}$ Fiocruz/CPqGM.

Introduction: About 257 million people are living with hepatitis B virus (HBV) infection worldwide, making this a global public health concern. In Brazil, seven HBV genotypes (A $\square \mathrm{G})$ were found circulating, but genotype A was the most prevalent (58.7\%) followed by genotypes D (23.4\%) and F (11.3\%). Oral fluid samples could be alternative specimens to determine HBV genotypes and to evaluate mutations associated to antiviral resistance.

Objective: This study aims to evaluate the usefulness of oral fluid samples to determine HBV genotype distribution, S/polymerase mutations and HBV subpopulation diversity among HBV chronically infected individuals.

Methodology: A total of 18 individuals gave serum and oral fluid samples. Informed consent was obtained from all participants prior to sample collection. Samples were submitted to PCR and nucleotide sequencing of HBV surface gene. Biochemical analysis of liver enzymes (ALT, AST, GGT) and HBV, $\mathrm{HCV}$ and HIV serological tests were also performed. MEGA 7.0 software was used to align and analyze nucleotide sequences and to reconstruct the phylogenetic tree using Maximum Likelihood method. Consensus sequences of each HBV isolate (serum and oral fluid) were submitted to a web-based software for subtyping and prediction of phenotypic resistance mutations in the polymerase gene (RT mutation) and to vaccine escape mutants analysis of gene S (Max-Planck-Institut f?r Informatik, Germany, at http:// hbv.geno2pheno.org/index.php).

Results: In this study, most of individuals were male (12/18; 66.7\%) and total mean age was $42.72 \pm$ 14.14 years. Among them, four individuals reported previous HBV treatment. All serum samples were $\mathrm{HBsAg}(+)$, anti-HBc(+) and anti-HBs(-); 55.6\% were $\mathrm{HBeAg}(+) /$ anti-HBe(-) and $11.1 \%$ were antiHIV $(+)$. Mean HBV-DNA viral load was $6.1 \pm 2.3 \mathrm{log} \mathrm{IU} / \mathrm{mL}$. HBV genotype distribution was: A $(72.2 \%)$, D (11.1\%), E (5.6\%) and F (11.1\%). A concordance of $100 \%$ in genotype classification and $99.8 \%$ of sequence similarity between paired oral fluid and serum was observed. It was possible to identify amino acid mutations in polymerase and/or S gene in all $18 \mathrm{HBV}$ serum and in all 10 oral fluid sequences. No antiviral primary resistance mutations were found. The most frequent detected polymorphisms in polymerase were N122H/Y, M129L V163I and I253V, that were observed in 12, 13, 11 and 9 serum samples, respectively, and in its paired oral fluid samples, when available. One or more escape mutations were detected in the $\mathrm{S}$ gene of five serum and four paired oral fluid samples. The mutation $\mathrm{Y} 100 \mathrm{C}$ was observed in two subjects.

Conclusion: This study demonstrated the accuracy of using oral fluid samples in tracking HBV mutations, genotyping and phylogenetic analysis what could be an important tool in molecular epidemiology studies with hard-to-reach populations.

Keywords: Hepatitis B virus; oral fluid; diagnosis 